\title{
Clinical characteristics and outcomes of patients with haematologic malignancies and COVID-19 suggest that prolonged SARS-CoV-2 carriage is an important issue
}

\author{
Robin Arcani ${ }^{1,2}(\mathbb{D})$ Julien Colle ${ }^{3} \cdot$ Raphaël Cauchois $^{1,2} \cdot$ Marie Koubi $^{1} \cdot$ Pierre-André Jarrot $^{1,2} \cdot$ Rodolphe Jean $^{1}$. \\ Arthur Boyer $^{4}$. Julie Lachamp ${ }^{4} \cdot$ Antoine Tichadou $^{3} \cdot$ Anne-Laure Couderc $^{5,6} \cdot$ Laure Farnault $^{3} \cdot$ Regis Costello $^{3}$. \\ Geoffroy Venton ${ }^{3}$. Gilles Kaplanski ${ }^{1,2}$
}

Received: 5 July 2021 / Accepted: 25 August 2021 / Published online: 13 September 2021

๑) The Author(s), under exclusive licence to Springer-Verlag GmbH Germany, part of Springer Nature 2021

\begin{abstract}
Specificities of COVID-19 disease course in patients with haematologic malignancies are still poorly studied. So, we aimed to compare patients with haematologic malignancies to patients without malignancies, matched by sex and age and hospitalised for COVID-19 at the same time and in the same centre. Among 25 patients with haematologic malignancies, we found that mortality ( $40 \%$ versus $4 \%, p<0.01)$, number of days with RT-PCR positivity $(21.2 \pm 15.9$ days [range, 3-57] versus $7.4 \pm 5.6$ days [range, 1-24], $p<0.01$ ), maximal viral load (mean minimal Ct, $17.2 \pm 5.2$ [range, 10-30] versus $26.5 \pm 5.1$ [range, 15-33], $p<0.0001$ ) and the delay between symptom onset and clinical worsening (mean time duration between symptom onset and first day of maximum requirement in inspired oxygen fraction, $14.3 \pm 10.7$ days versus $9.6 \pm 3.7$ days, $p=0.0485$ ) were higher than in other patients. COVID-19 course in patients with haematologic malignancies has a delayed onset and is more severe with a higher mortality, and patients may be considered as superspreaders. Clinicians and intensivists need to be trained to understand the specificity of COVID-19 courses in patients with haematological malignancies.
\end{abstract}

Keywords COVID-19 $\cdot$ SARS-CoV-2 $\cdot$ Haematologic malignancies $\cdot$ Mortality $\cdot$ Super-spreader

Robin Arcani

robin.arcani@ap-hm.fr

1 Department of Internal Medicine and Clinical Immunology, CHU La Conception, Assistance Publique-Hôpitaux de Marseille (AP-HM), 145 Boulevard Baille, Marseille 13005, France

2 Center for Cardiovascular and Nutrition Research (C2VN), INRA 1260, INSERM UMR_S 1263, Aix-Marseille University, Marseille, France

3 Hematology and Cellular Therapy Department, CHU La Conception, Assistance Publique-Hôpitaux de Marseille (AP-HM), Marseille, France

4 Réanimation Des Brûlés, CHU La Conception, Assistance Publique-Hôpitaux de Marseille (AP-HM), Marseille, France

5 Geriatric and Therapeutic Unit, Internal Medicine, CHU Sainte Marguerite, Assistance Publique-Hôpitaux de Marseille (AP-HM), Marseille, France

6 Etablissement Français du Sang, Anthropologie, Droit, Ethique en Santé, Centre National de La Recherche Scientifique (CNRS), Marseille, France

\section{Introduction}

Since December 2019, an outbreak of a previously unknown coronavirus, named severe acute respiratory syndrome coronavirus 2 (SARS-CoV-2), has spread around the world, inducing a disease called COVID-19 by the World Health Organization (WHO) in February 2020. COVID-19 has caused a pandemic with more than 70 million cases of SARS-CoV-2 infections worldwide in December 2020, along with consecutive mortality, especially in populations with comorbidities [1]. Patients with haematologic malignancies may exhibit a higher incidence of severe and lethal forms of COVID-19 [2] than other patients [3]. Few studies, however, precisely compared COVID19 evolution in patients with and without underlying haematologic malignancies, treated in the same centre by the same medical team [4]. We report our experience in taking care of such 2 groups of patients 
during the fall 2020 and compared 2 matched populations of patients.

\section{Material and methods}

We retrospectively included all consecutive adult patients (aged $\geq 18$ years) admitted to our hospital (La Conception, University Hospital of Marseille, France) between September and November 2020. Inclusion criteria were a WHO-defined haematologic malignancy and laboratoryconfirmed SARS-CoV-2 infection assessed by RT-PCR on nasopharyngeal swabs. The control cohort consisted of a 1:1 sex- and age-matched randomised patients (from the medical database of our hospital) with symptomatic and laboratory-confirmed SARS-CoV-2 infection assessed by RT-PCR on nasopharyngeal samples but without haematologic malignancy admitted into the same hospital, between September and November 2020. During the SARS-CoV-2 pandemic, all patients were followed by the same medical team and, when necessary, were transferred into the same intensive care unit (ICU). Clinical, biological and follow-up data of these patients were collected from electronic medical records. We registered day 1 of oxygen supplementation as $\mathrm{D} \mathrm{O}_{2}$. The first day of maximum requirement in inspired oxygen fraction during hospitalisation was considered $\mathrm{D} 1 \mathrm{maxO}_{2}$. The number of days of viral positivity was defined as those comprised between the first positive RT-PCR test and subsequent negative test. Viral load was analysed by quantitative RT-PCR from nasopharyngeal swabs. Negative results for viral RNA detection were defined as a cycle threshold $(\mathrm{Ct})$ value $\geq 35$. Patients underwent a low-dose chest computed tomography (CT) and were classified into five grades (minimal, moderate, intermediate, severe and critical) based on the extent of lesions in the pulmonary parenchyma $(<10 \%, 10-25 \%, 25-50 \%$, $50-75 \%,>75 \%$, respectively).

This study was approved by the Institutional Review Board of Assistance Publique - Hôpitaux de Marseille (GDPR number PADS21-4). The study was conducted according to the Declaration of Helsinki.

Quantitative variables were described using means and standard deviation (SD) or range; categorical variables were described using numbers and percentages. Quantitative data were compared using the Student's $t$ or Mann-Whitney $U$ test, while qualitative data were compared with the Chi-square or Fisher's exact test when appropriate. The tests were two-sided. $p$ values $<0.05$ were considered significant. All analyses were performed with $\mathrm{R}$ software (R Foundation for Statistical Computing, Vienna, Austria).

\section{Results}

COVID-19 was diagnosed in 25 patients with haematologic malignancies, 19 male patients, mean age $64.8 \pm 16.0$ years (range, 20-93), with a mean follow-up duration of $43.3 \pm 31.6$ days (range, $4-102$ ). Eight patients had acute myeloid leukaemia, 3 acute lymphoid leukaemia, 5 diffuse large B cell lymphoma, 3 low-grade lymphoma, 1 Hodgkin's lymphoma, 1 chronic lymphoid leukaemia and 4 multiple myeloma. Twenty-three patients were given systemic anti-cancer therapy at the time of COVID-19 diagnosis: particularly, eight patients were treated with rituximab and one with daratumumab. The most frequent comorbidities included undernutrition (64\%), hypertension (40\%), cardiovascular disease $(32 \%)$, dyslipidaemia $(28 \%)$, diabetes $(20 \%)$, current smoking (20\%), chronic lung disease $(16 \%)$ and chronic kidney failure (4\%). Twenty-one patients were symptomatic, while 16 needed oxygen and 5 patients developed acute respiratory distress syndrome (ARDS) requiring mechanical ventilation. At the admission, 8 patients were neutropenic, and 21 patients were lymphocytopenic. Lung CT showed COVID-19 typical lesions in 17 of 19 patients, consisting in minimal, moderate, intermediate, severe and critical lesions in $31.6 \%$, $10.5 \%, 26.3 \%, 15.8 \%$ and $5.3 \%$, respectively. Twenty-four patients received treatment for COVID-19: 80\% empirical antibiotics, 76\% heparin, 36\% dexamethasone, $28 \%$ and $16 \%$, respectively, received off-label anakinra and ruxolitinib, $12 \%$ hydroxychloroquine and $8 \%$ remdesivir. The mean time for oxygen supplementation was $11.2 \pm 13.6$ days (range, $0-48$ ). Two patients developed a documented pneumonia (one due to Streptococcus pneumoniae and one due to Klebsiella aerogenes) from the lower respiratory tract specimens. Two patients developed a bacteraemia (one due to Staphylococcus hominis and one due to Pseudomonas aeruginosa). Nine patients were admitted into the ICU. Overall, the 28-day mortality rate was $40 \%$. The control cohort consisted in 25 patients (19 male patients; mean age, $66.7 \pm 11.1$ years; range, 47-91; $p>0.05$ ) (Table 1). All patients needed oxygen supplementation, but only one patient developed ARDS and received mechanical ventilation. Lung CT showed COVID-19 typical lesions in 25 patients, consisting in minimal, moderate, intermediate, severe and critical lesions in $4 \%, 48 \%, 40 \%, 8 \%$ and $0 \%$, respectively. Six patients required admission into the ICU, and the 28-day mortality was $4 \%(p<0.01)$ (Fig. 1$)$. At the admission, no patients were neutropenic, and 21 patients were lymphocytopenic. The SARS-CoV-2 strain was the original strain from Wuhan in all patients. The mean number of days of viral positivity $(21.2 \pm 15.9$ days [range, $3-57$ ] versus $7.4 \pm 5.6$ days [range, $1-24$ ], $p<0.01)$ and the 
Table 1 Characteristics of the patients

\begin{tabular}{ll}
\hline Characteristics & $\begin{array}{l}\text { Patient } \\
\text { malign }\end{array}$ \\
\hline Age (years) $^{\mathrm{a}}$ & $64.8 \pm$ \\
Male gender $^{\mathrm{b}}$ & $19(76)$ \\
Haematologic cancer $^{\mathrm{b}}$ & \\
- AML & $8(32)$ \\
- ALL & $3(12)$ \\
- DLBCL & $5(20)$ \\
- Hodgkin lymphoma & $1(4)$ \\
- Low-grade lymphoma & $3(12)$ \\
- CLL & $1(4)$ \\
- MM & $4(16)$
\end{tabular}

Number of oncologic treatment lines ${ }^{\mathrm{b}}$

$\begin{array}{ll}-0 & 2(8) \\ -1 & 17(68) \\ -2 & 5(20) \\ -3 & 1(4)\end{array}$

Comorbidities $^{\mathrm{b}}$

- Undernutrition $16(64)$

- Hypertension $10(40)$

- Cardiovascular disease 8 (32)

- Dyslipidaemia $7(28)$

- Diabetes $5(20)$

- Currently smoking $5(20)$

- Chronic lung disease 4 (16)

- Chronic kidney failure 1 (4)

Symptoms ${ }^{\text {b }}$

$21(84)$

$16(64)$

$15(60)$

$5(20)$

$17.2 \pm 5.2$

$21.2 \pm 15.9$

$0(0)$

$11(44)$

4 (16)

$8(32)$

$14(56)$

$2(8)$

$4(16)$

3 (12)

NS

19 (76)

NS

- Oxygen need

- ARDS

Minimal $\mathrm{Ct}^{\mathrm{a}}$

Days of RT-PCR positivity ${ }^{a}$

Grade of lung involvement ${ }^{\mathrm{b}}$

- No lesion

$2(10.5)$

$6(31.6)$

$2(10.5)$

- Moderate

$5(26.3)$

$3(15.8)$

- Severe

- Critical

$1(5.3)$

25 (100)

25 (100)

18 (72)

1 (4)

$26.5 \pm 5.1$

$<0.0001$

$7.4 \pm 5.6$

$<0.01$

COVID-19 management ${ }^{\mathrm{b}}$

- Heparins

19 (76)

$20(80)$

$10(40)$

- AZT

9 (36)

- Dexamethasone

$7(28)$
$4(16)$

- Anakinra

$3(12)$

- HCQ

- Remdesivir

2 (8)

$11.2 \pm 13.6$

$0(0)$

1 (4)

12 (48)

10 (40)

$2(8)$

$0(0)$

$<0.001$

NS

NS

NS

0.019

NS

NS

NS

Oxygen duration ${ }^{\mathrm{a}}$

5 (20)

9 (36)

25 (100)

25 (100)

24 (96)

25 (100)

1 (4)

3 (12)

8 (32)

$0(0)$

NS

0.032

0.01

NS

NS

NS

Mechanical ventilation

$9.9 \pm 4.9$

0.022

NS

$<0.0001$

$<0.0001$

0.049

NS

NS

NS

NS

1 (4)

NS

6 (24)

NS

Death $^{\mathrm{b}}$

$10(40)$
$<0.001$

${ }^{\mathrm{a}}$ mean \pm standard deviation

${ }^{\mathrm{b}} n(\%)$

$N S$, non-significant; $A M L$, acute myeloid leukaemia; $A L L$, acute lymphoid leukaemia; $D L B C L$, diffuse large B cell lymphoma; $C L L$, chronic lymphoid leukaemia; $M M$, multiple myeloma; $A R D S$, acute respiratory distress syndrome; $C t$, cycle threshold; $A Z T$, azithromycin; $H C Q$, hydroxychloroquine; $I C U$, intensive care unit 
Fig. 1 A 28-day mortality in COVID-19 patients

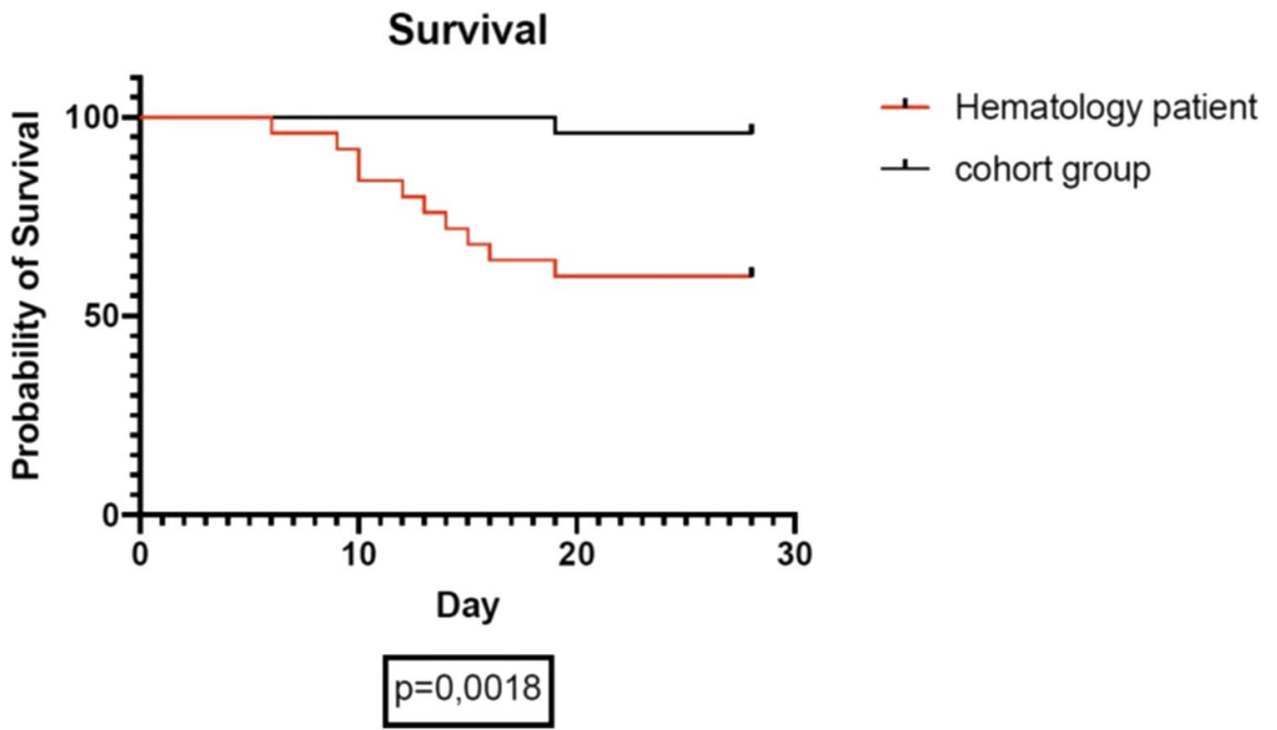

maximal viral load on a nasopharyngeal sample (mean minimal Ct, $17.2 \pm 5.2$ [range, $10-30$ ] versus $26.5 \pm 5.1$ [range, 15-33], $p<0.0001$ ) were higher in patients with haematologic malignancy than in the control cohort. The mean time duration between the first positive RT-PCR and $\mathrm{D} 1 \mathrm{O}_{2}$ and the first positive RT-PCR and D1maxO was higher in onco-haematologic patients $(7.2 \pm 5.7$ versus $3.6 \pm 3.5$ days, $p=0.0011$, and $15.3 \pm 10.9$ versus $6.2 \pm 4.3$ days, $p=0.0022$, respectively). There was no difference in the delay between symptom onset and $\mathrm{D}_{1} \mathrm{O}_{2}$ in both groups of patients (6.1 days versus 6.3 days, $p>0.05$ ). However, the mean time duration between symptom onset and $\mathrm{D} 1 \mathrm{maxO}_{2}$ was higher in patients with haematologic cancer than in the control cohort (14.3 \pm 10.7 days versus $9.6 \pm 3.7$ days, $p=0.0485$ ) (Supplemental Fig. 1). ARDS was the only factor associated with death in patients with haematologic malignancies ( $40 \%$ versus $4 \%, p=0.0412$ ). Anakinra was more frequently used in patients who died (50\% versus $13.3 \%, p=0.0455)$. There were only three surviving patients $(33.3 \%)$ out of the nine with haematologic malignancies transferred into the ICU.

\section{Discussion}

Few studies compared COVID-19 patients with and without haematologic malignancy, followed and treated by the same medical team in the same hospital over the same period of time. Our study fulfils these requirements, therefore avoiding bias due to differences in patients care given by different physician teams over different periods of time. Nevertheless, in our retrospective work, the 2 groups were not strictly comparable. Particularly, patients without haematologic malignancies were more frequently treated with dexamethasone than patients with haematologic neoplasm. That was due to the prolonged viral phase in patients with haematologic malignancies; they were not good candidates to receive immunosuppressive drug (according to the treatment protocol of our hospital, patients were given dexamethasone when the viral phase was about to end, when $\mathrm{Ct}$ was $\geq 30$ ). Despite limitations due to the limited number of patients, our results clearly confirm the higher mortality rate observed in COVID-19 patients with haematologic malignancies [3-6] and highlight the fact that clinical symptoms may worsen later, beyond the 8-10 days of disease course, in these patients [7]. Compared to the reported 3 stages of COVID-19 [8], haematologic patients appeared to have a longer duration of the viral phase (stages 1 and 2A) which may induce a delayed hyperinflammation phase (stage 3 ) and a delayed ( $\sim 12-20$ days) clinical deterioration, thus requiring longer monitoring. In addition, prolonged viral persistence in the nasopharynx of haematologic patients may have significant implications for management. First, the prolonged SARS-CoV-2 carriage may complicate the treatment of the viral-induced inflammatory phase. Indeed, the use of steroids or other immunomodulating agents, such as anakinra, in the absence of an effective antiviral therapy may in fact favour the viral disease. The use of convalescent plasma or antiviral antibodies to decrease the viral load may thus be absolutely required, especially when immunomodulation is considered in these patients. Moreover, uncontrolled prolonged viral disease may delay chemotherapy treatments, resulting in cancer progression. Second, during this viral period, haematologic patients presented a higher viral load resulting in possible increased contagiousness which may increase the likelihood of nosocomial epidemic clusters, notably in onco-haematology departments. Worse outcomes, longer viral period and higher viral load in haematologic 
patients should then encourage to aggressively prevent COVID-19, in a protective environment and to quickly isolate suspected patients from other haematologic patients. Third, it may favour the emergence of new viral variants, as RNA viruses are characterised by a high mutation rate proportional to the carriage time duration, constituting an important epidemiologic issue $[9,10]$. Therefore, the specific aspects of COVID-19 in patients with haematologic malignancies may lead to specific epidemiologic measures and therapies.

Supplementary Information The online version contains supplementary material available at https://doi.org/10.1007/s00277-021-04656-z.

Author contribution RA, JC, RC, MK, GV and GK made substantial contributions to the conception or design of the work and drafted the work.

PAJ, RJ, AB, JL, AT, ALC, LF and RC critically revised the work.

All the authors made substantial contributions to the analysis and interpretation of data, approved the version to be published and agreed to be accountable for all aspects of the work in ensuring that questions related to the accuracy or integrity of any part of the work are appropriately investigated and resolved.

Data availability Available on reasonable request.

\section{Declarations}

Ethics approval This research study was conducted retrospectively from data obtained for clinical purposes. This study was approved by the Institutional Review Board of Assistance Publique - Hôpitaux de Marseille (GDPR number PADS21-4). The study was conducted according to the Declaration of Helsinki.

Consent to participate Not applicable.

Consent for publication Not applicable.

Conflict of interest The authors declare no competing interests.

\section{References}

1. COVID-19 pandemic. In: European Centre for Disease Prevention and Control. https://www.ecdc.europa.eu/en/covid-19-pandemic. Accessed 24 Dec 2020

2. Vijenthira A, Gong IY, Fox TA et al (2020) Outcomes of patients with hematologic malignancies and COVID-19: a systematic review and meta-analysis of 3377 patients. Blood. https://doi.org/ 10.1182/blood.2020008824

3. Passamonti F, Cattaneo C, Arcaini L et al (2020) Clinical characteristics and risk factors associated with COVID-19 severity in patients with haematological malignancies in Italy: a retrospective, multicentre, cohort study. Lancet Haematol 7:e737-e745. https://doi.org/10.1016/S2352-3026(20)30251-9

4. Shah V, Ko Ko T, Zuckerman M et al (2020) Poor outcome and prolonged persistence of SARS-CoV-2 RNA in COVID-19 patients with haematological malignancies; King's College Hospital experience. Br J Haematol 190:e279-e282. https://doi.org/ 10.1111/bjh.16935

5. Martínez-López J, Mateos M-V, Encinas C et al (2020) Multiple myeloma and SARS-CoV-2 infection: clinical characteristics and prognostic factors of inpatient mortality. Blood Cancer J 10:103. https://doi.org/10.1038/s41408-020-00372-5

6. Cattaneo C, Daffini R, Pagani C et al (2020) Clinical characteristics and risk factors for mortality in hematologic patients affected by COVID-19. Cancer 126:5069-5076. https://doi.org/10.1002/ cncr.33160

7. Hu B, Guo H, Zhou P, Shi Z-L (2020) Characteristics of SARSCoV-2 and COVID-19. Nat Rev Microbiol 1-14.https://doi.org/ 10.1038/s41579-020-00459-7

8. Siddiqi HK, Mehra MR (2020) COVID-19 illness in native and immunosuppressed states: a clinical-therapeutic staging proposal. J Heart Lung Transplant 39:405-407. https://doi.org/10.1016/j. healun.2020.03.012

9. Manrubia SC, Escarmís C, Domingo E, Lázaro E (2005) High mutation rates, bottlenecks, and robustness of RNA viral quasispecies. Gene 347:273-282. https://doi.org/10.1016/j.gene.2004.12. 033

10. Martinot M, Jary A, Fafi-Kremer S et al (2020) Remdesivir failure with SARS-CoV-2 RNA-dependent RNA-polymerase mutation in a B-cell immunodeficient patient with protracted Covid-19. Clin Infect Dis. https://doi.org/10.1093/cid/ciaa1474

Publisher's note Springer Nature remains neutral with regard to jurisdictional claims in published maps and institutional affiliations. 